\title{
Púrpura trombocitopênica trombótica - remissão completa em paciente com mau prognóstico após tratamento com plasmaférese terapêutica e rituximabe
}

\author{
Successful outcome in poor-prognostic acute thrombotic thrombocytopenic purpura treated with \\ plasma exchange and rituximab
}

Cesar de Almeida Neto ${ }^{1}$

Mirianceli C. Mendonça ${ }^{2}$

Marcelo C. Braga ${ }^{2}$

Fabiana S. Ghaname ${ }^{2}$

Fernanda O. S. Gobette ${ }^{3}$

Verônica S. P. Pires ${ }^{4}$

Anis Ganme

Jorge N. Ghaname
A púrpura trombocitopênica trombótica (PTT) é uma doença rara e fatal que deve ser diagnosticada e tratada prontamente a fim de se obter melhor resposta terapêutica. Apresentamos um caso de PTT aguda grave tratada com plasmaférese e rituximabe. Ao diagnóstico, a paciente apresentava anemia hemolítica microangiopática, icterícia, febre, convulsões, seguidas por coma e choque hipovolêmico. Os exames laboratoriais iniciais mostravam $\mathrm{DHL}=2.860 \mathrm{IU} / \mathrm{L}$, contagem de plaquetas de $37 \times 10^{9} / \mathrm{L}$, hemoglobina de 5,1 g/dL e no esfregaço de sangue periférico havia a presença de esquizócitos. Iniciado tratamento para PTT com pulsoterapia com metilprednisolona e plasmaféreses terapêuticas diárias com troca de uma volemia plasmática e substituição com plasma fresco congelado. Após cinco sessões de plasmaférese, houve piora no quadro neurológico, acompanhado por aumento importante de DHL, ALT, AST e a contagem de plaquetas era de $72 \times 10^{9} / \mathrm{L}$. Iniciamos o uso de rituximabe na dose padrão de $375 \mathrm{mg} / \mathrm{m}^{2} / \mathrm{semana} / 4$ semanas e passamos a utilizar plasma pobre em crioprecipitado como reposição durante as plasmaféreses. Dois dias após a mudança na conduta terapêutica, houve importante melhora do quadro neurológico, estabilização da contagem de plaquetas e queda acentuada de DHL. Após 23 procedimentos de plasmaférese e quatro doses de rituximabe, a paciente apresentou remissão completa, mantida há 34 meses. A plasmaférese terapêutica com plasma pobre em crioprecipitado e o uso concomitante de rituximabe foi uma estratégia útil no tratamento deste caso de PTT aguda grave. Porém, ensaios clínicos prospectivos e randomizados são necessários para confirmar estes achados. Rev. bras. hematol. hemoter. 2008;30(1):71-74.

Palavras-chave: Púrpura trombocitopênica trombótica; troca de plasma; rituximabe; anemia hemolítica; terapêutica.

\section{Introdução}

A púrpura trombocitopênica trombótica (PTT) é uma doença rara e fatal que deve ser diagnosticada e tratada prontamente a fim de se obter melhor resposta terapêutica. A incidência aproximada é de 3,7 casos para 1.000.000 habitantes. As mulheres são mais freqüentemente afetadas do que os homens com uma incidência quase duas vezes superior. ${ }^{1}$ Clinicamente caracteriza-se por uma pêntade clássica constituída por anemia hemolítica microangiopática, trombocitopenia, alterações neurológicas, insuficiência renal e febre. ${ }^{2}$ Estes sintomas estão relacionados à presença de trombos ricos em plaquetas e fator de von Willebrand na microvasculatura do organismo e associados, na maioria das vezes, com a defici-

${ }^{1}$ Doutor em Hematologia pela FMUSP. Médico assistente do Serviço de Hemoterapia do Hospital Nove de Julho.

${ }^{2}$ Médica(o) assistente do Serviço de Hemoterapia do Hospital Nove de Julho.

${ }^{3}$ Médica assistente do Serviço de Hematologia do Hospital Nove de Julho.

${ }^{4}$ Enfermeira do Serviço de Hemoterapia do Hospital Nove de Julho.

${ }^{5}$ Diretor do Serviço de Hemoterapia do Hospital Nove de Julho.

Correspondência: Cesar de Almeida Neto

Rua Peixoto Gomide, 625 - $7^{\circ}$ andar - Bela Vista

01409-902 - São Paulo-SP - Brasil

Tel.: (11) 3285-2922 - Fax: (11) 3284-4426

E-mail: cesarnt@uol.com.br 
ência funcional grave da ADAMTS,13 uma metaloprotease plasmática clivadora do fator de von Willebrand. A deficiência da ADAMTS13 pode ser congênita, secundária à mutação do gene desta enzima, na forma rara denominada Síndrome de Upshaw-Schulman ou mais comumente devido à presença de auto-anticorpos circulantes anti-ADAMTS13. ${ }^{3}$

O uso da plasmaférese diminuiu a mortalidade da PTT de $90 \%$ para $10 \%$ a 30\%, ${ }^{4}$ devido à correção da deficiência da ADATMS13, bem como remoção de auto-anticorpos patogênicos e citocinas endotélio-estimulantes. ${ }^{2}$ Porém, em casos agudos graves, refratários, ou quando há recaídas, terapêuticas adicionais, tais como corticosteróides, ciclofosfamida, azatioprina, imunoglobulinas em altas doses e esplenectomia podem ser necessárias.

Tendo em vista que muitos casos de PTT idiopática na verdade apresentam uma etiologia auto-imune, novos tratamentos imunossupressores podem ser efetivos na remissão da doença, evitando plasmaféreses a longo prazo e diminuindo a morbidade e mortalidade da doença.

Apresentamos um caso de PTT aguda grave tratada com plasmaférese e rituximabe.

\section{Relato de Caso}

Paciente do sexo feminino, 44 anos, internada na CTI com quadro grave de anemia hemolítica microangiopática, icterícia, febre, convulsões seguidas por coma e choque hipovolêmico. Seus familiares relataram que há vinte dias ela havia apresentado dores nas articulações do joelho e lesões eritematosas em pernas e tronco. Na ocasião apresentou FAN positivo (1/160). Uma semana antes da internação notaram-se alterações no comportamento da paciente. O DHL era de $2.860 \mathrm{IUI} / \mathrm{L}$, contagem de plaquetas de $37 \times 10^{9} / \mathrm{L}$, hemoglobina de 5,1 g/dL e no esfregaço de sangue periférico havia a presença de esquizócitos. AST era de $240 \mathrm{U} / \mathrm{L}$, ALT de $166 \mathrm{U} / \mathrm{L}$, a sorologia para HIV e hepatites B e C eram negativas e a tomografia de crânio e ressonância nuclear magnética de encéfalo normais. Diagnosticou-se PTT e iniciado pulsoterapia com metilprednisolona $1 \mathrm{~g} / \mathrm{dia} / 3$ dias e plasmaférese terapêutica de uma volemia plasmática diária com plasma fresco congelado. Após duas sessões houve uma discreta melhora do quadro neurológico, a contagem de plaquetas subiu para 95 x 10\%/L e o nível de DHL caiu para 2.199 UI/L. Após cinco sessões de plasmaférese, houve piora do quadro neurológico, aumento importante da DHL (7.139 U/L), ALT (1.700 U/L), AST (3.000 $\mathrm{U} / \mathrm{L})$ e a contagem de plaquetas caiu para $72 \times 10^{9} / \mathrm{L}$. Iniciamos o uso de rituximabe na dose padrão de $375 \mathrm{mg} / \mathrm{m}^{2} / \mathrm{sema-}$ na/4 semanas e passamos a utilizar plasma pobre em crioprecipitado como reposição durante as plasmaféreses. Dois dias após a mudança na conduta terapêutica houve importante melhora do quadro neurológico, estabilização da contagem de plaquetas (Figura 1) e queda acentuada da DHL (Figura 2). Após 23 procedimentos de plasmaférese e quatro

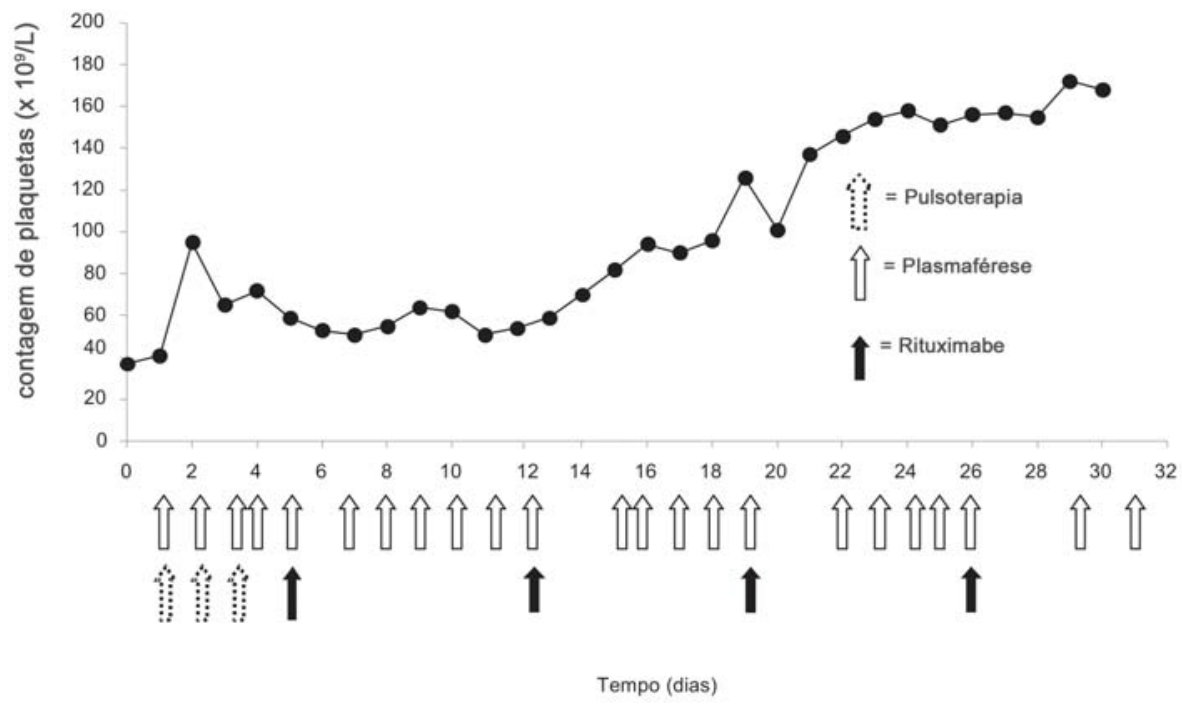

Figura 1. Evolução na contagem de plaquetas no decorrer do tratamento com pulsoterapia, plasmaférese terapêutica e rituximabe

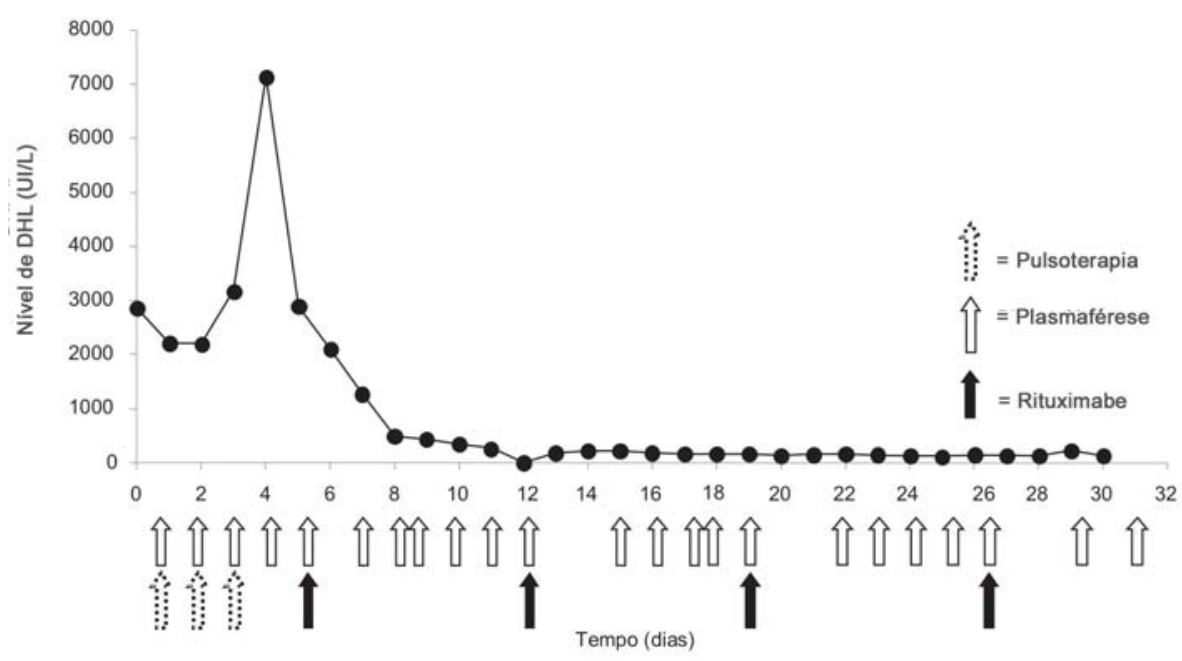

Figura 2. Níveis de DHL ao diagnóstico, durante e após tratamento com pulsoterapia, plasmaférese terapêutica e rituximabe 
doses de rituximabe, a paciente alcançou remissão completa. Ocorreram três episódios de urticária moderada durante as sessões de plasmaférese, que melhoraram após uso de cloridrato de difenidramina e hidrocortisona. Não houve efeitos tóxicos observados durante e após o uso de rituximabe. A paciente permanece em remissão completa da doença há 34 meses.

\section{Discussão}

Em pacientes com PTT refratária à plasmaférese, várias terapêuticas vêm sendo empregadas a fim de diminuir a mortalidade e a morbidade. No caso apresentado, a introdução precoce de rituximabe associado à plasmaférese com reposição de plasma pobre em crioprecipitado foi efetiva para induzir a remissão completa e sustentada da doença.

Embora a plasmaférese terapêutica associada ao uso de corticosteróides como tratamento de primeira linha da PTT tenha em muito aumentado a sobrevida destes pacientes, há um subgrupo que não alcança resposta completa sendo considerados refratários. Por definição, PTT refratária consiste em trombocitopenia persistente (contagem de plaquetas $<150 \times 10^{9} / \mathrm{L}$ ) ou elevação do DHL após um total de sete trocas plasmáticas diárias de uma volemia. ${ }^{5}$ Porém, a demora em iniciar-se o tratamento da PTT, presença de estupor ou coma e creatinina elevada são preditores de falha do tratamento e aumento da mortalidade. ${ }^{6}$ Nestes casos, uma abordagem mais agressiva, semelhante à doença refratária é recomendada. Uma das alternativas propostas é a realização de plasmaféreses terapêuticas e reposição com plasma pobre em crioprecipitado, que não contém os multímeros do fator de Von Willebrand e poderia trazer benefícios clínicos. ${ }^{7}$ Outra alternativa é a intensificação dos procedimentos de plasmaférese, ou seja, dobrar a volemia durante as trocas plasmáticas ou realizar a troca de uma volemia plasmática a cada 12 horas. ${ }^{5} \mathrm{~A}$ intensificação é uma medida empírica e somente deve ser considerada em casos de risco de morte.

Nossa paciente apresentava prognóstico reservado ao diagnóstico, caracterizado pelo coma, e apresentou piora clínica e laboratorial após a troca de cinco volemias plasmáticas mais pulsoterapia com corticosteróides. Portanto, optamos por realizar as trocas plasmáticas com reposição de plasma pobre em crioprecipitado e iniciamos a administração de rituximabe.

Há cerca de vinte artigos relatando o tratamento de 37 pacientes com rituximabe na PTT, ${ }^{8}$ com benefícios descritos em 25 (93\%). Entretanto, em somente um estudo, rituximabe foi administrado no primeiro episódio e na fase aguda da doença. Fakhouri et al. ${ }^{9}$ trataram seis pacientes com PTT refratária à plasmaférese terapêutica com quatro doses semanais de rituximabe. Dois deles estavam no primeiro episódio da doença, em plasmaférese por um mês e, adicionalmente, haviam recebido vincristina. A remissão com- pleta foi obtida em todos os pacientes entre 5 e 14 dias após a quarta dose de rituximabe.

Importante notar que embora a remoção do rituximabe durante a plasmaférese seja motivo de preocupação, não há relatos referentes aos níveis deste agente em pacientes durante estes procedimentos. Além disso, não se sabe se a plasmaférese tem efeito imediato ou tardio nas doses terapêuticas de rituximabe. Embora ainda seja motivo de controvérsia, acredita-se que, devido à rápida distribuição intravascular do anticorpo monoclonal, os procedimentos de plasmaférese, um ou mais dias após administração do medicamento, não devam afetar os níveis terapêuticos do medicamento. Até que mais estudos sobre este aspecto estejam disponíveis, recomenda-se adiar os procedimentos de plasmaférese pelo maior tempo possível. Porém, nos casos em que a plasmaférese não pode ser postergada, o procedimento deve ser realizado. ${ }^{10}$

Anteriormente ao diagnóstico de PTT, a paciente apresentou quadro clínico sugestivo de lúpus eritematoso sistêmico (LES), cujo diagnóstico não se confirmou posteriormente. O tratamento do LES, uma das causas de PTT secundária, pode ser realizado através de plasmaférese terapêutica ou da administração de rituximabe. Entretanto, a plasmaférese terapêutica tem indicação restrita nestes ca$\operatorname{sos}^{11}$ e o uso de rituximabe foi descrito em pouco mais de cem pacientes em estudos fase II. ${ }^{12,13}$ Embora não seja possível afastar quadro de LES que se iniciou com PTT, devido à resposta clínica apresentada pela paciente e à falta de evidências de LES após remissão prolongada, acreditamos que este caso seja mais compatível com PTT auto-imune primária.

Uma limitação que encontramos foi a impossibilidade de monitorar os níveis de atividade da ADAMTS13 e a presença de anticorpos anti-ADAMTS 13 plasmáticas. No estudo de Fakhouri et al., ${ }^{9}$ todos os pacientes que obtiveram remissão não mais apresentaram anticorpos anti-ADAMTS13 plasmáticos detectáveis e houve recuperação entre $29 \%$ a $75 \%$ da atividade desta enzima no plasma entre sete a 24 semanas após a infusão da última dose de rituximabe. Tendo em vista que a depleção de células B pelo rituximabe dura de seis a nove meses com recuperação total dos níveis prétratamento em 12 meses, a monitoração da atividade da ADAMTS13 assim como o reaparecimento de anticorpos antiADAMTS13 poderiam ser indícios de recaída da doença e da necessidade de tratamento de manutenção precoce.

Concluindo, o uso de plasmaférese terapêutica com plasma pobre em crioprecipitado associado à introdução precoce de rituximabe foi efetivo na obtenção de remissão completa prolongada nesta paciente com quadro agudo e grave de PTT. Estudos clínicos multicêntricos e randomizados são necessários para esclarecer qual a melhor estratégia para reposição plasmática nas plasmaféreses terapêuticas e ainda o papel do rituximabe no tratamento de primeira linha da PTT. 


\begin{abstract}
Thrombotic thrombocytopenic purpura (TTP) is a rare severe disease that must be diagnosed and treated promptly for a successful outcome. We report a case of severe acute TTP treated with plasma exchange and rituximab. The patient presented at diagnosis with severe microangiopathic haemolytic anemia, jaundice, fever, seizures followed by coma and hypovolemic shock. Her LDH was 2,860 IU/L; platelet count $37 \times 10^{9} / \mathrm{L}$; hemoglobin $5.1 \mathrm{~g} / \mathrm{dL}$; blood smears contained schistocytes. Treatment for TTP was started with pulses of methylprednisolene and daily single-volume plasma exchange using fresh frozen plasma. After five plasma exchanges, the neurological status worsened, $L D H$ increased sharply as did ALT and AST and the platelet count dropped to 72 x 10\%/ L. Rituximab (weekly doses of $375 \mathrm{mg} / \mathrm{m}^{2}$ for 4 weeks) and plasma exchange with cryosupernatant substitution fluid were initiated. Within two days there was neurological improvement, stable platelet levels and decreased LDH. Complete remission, achieved after 23 plasma exchanges and four doses of rituximab, has been maintained for 34 months. Plasma exchange with cryosupernatant substitution fluid and rituximab played an important role in the successful treatment of this patient with severe acute TTP. A systematic clinical trial should be considered in order to confirm these findings. Rev. bras. hematol. hemoter. 2008;30 (1):71-74.
\end{abstract}

Key words: Thrombotic thrombocytopenic purpura; plasma exchange; rituximab; hemolytic anemia; therapy.

\section{Agradecimentos}

Os autores agradecem a cuidadosa revisão e sugestões apresentadas pelo Dr. Marcelo de Medeiros Pinheiro, assistente doutor da Disciplina de Reumatologia da Unifesp e a colaboração da Dra. Maria Cristina Bezerra para que esta revisão fosse efetivada.

\section{Referências Bibliográficas}

1. Torok TJ, Holman RC, Chorba TL. Increasing mortality from thrombotic thrombocytopenic purpura in the United States analysis of national mortality data, 1968-1991. Am J Hematol. 1995;50(2):84-90.

2. Sadler JE, Moake JL, Miyata T, George JN. Recent advances in thrombotic thrombocytopenic purpura. Hematology Am Soc Hematol Educ Program. 2004;407-23.

3. Tsai HM. Advances in the pathogenesis, diagnosis, and treatment of thrombotic thrombocytopenic purpura. J Am Soc Nephrol. 2003; 14(4):1072-81.

4. Rock GA, Shumak KH, Buskard NA, Blanchette VS, Kelton JG, Nair RC et al. Comparison of plasma exchange with plasma infusion in the treatment of 'thrombotic thrombocytopenic purpura. Canadian Apheresis Study Group. N Engl J Med. 1991;325(6):393-7.

5. Allford SL, Hunt BJ, Rose P, Machin SJ; Haemostasis and Thrombosis Task Force, British Committee for Standards in Haematology. Guidelines on the diagnosis and management of the thrombotic microangiopathic haemolytic anaemias. Br J Haematol 2003; 120 (4):556-73.
6. Pereira A, Mazzara R, Monteagudo J, Sanz C, Puig L, Martinez A, et al. Thrombotic thrombocytopenic purpura/hemolytic uremic syndrome: a multivariate analysis of factors predicting the response to plasma exchange. Ann Hematol. 1995;70(6):319-23.

7. Molinari E, Costamagna L, Perotti C, Isernia P, Pagani A, Salvaneschi L. Refractory thrombotic thrombocytopenic purpura: successful treatment by plasmapheresis with plasma cryosupernatant. Haematologica. 1993;78(6):389-92.

8. George JN, Woodson RD, Kiss JE, Kojouri K, Vesely SK. Rituximab therapy for thrombotic thrombocytopenic purpura: a proposed study of the Transfusion Medicine/Hemostasis Clinical Trials Network with a systematic review of rituximab therapy for immunemediated disorders. J Clin Apher. 2006;21(1):49-56.

9. Fakhouri F, Vernant JP, Veyradier A, Wolf M, Kaplanski G, Binaut $\mathrm{R}$ et al. Efficiency of curative and prophylactic treatment with rituximab in ADAMTS13-deficient thrombotic thrombocytopenic purpura: a study of 11 cases. Blood. 2005;106(6):1932-7.

10. Yomtovian R, Niklinski W, Silver B, Sarode R, Tsai HM. Rituximab for chronic recurring thrombotic thrombocytopenic purpura: a case report and review of the literature. Br J Haematol. 2004; 124(6):787-95.

11. Smith JW, Weinstein R, Hillyer KL. Therapeutic apheresis: a summary of current indication categories endorsed by the AABB and the American Society for Apheresis. Transfusion. 2003;43:820-2.

12. Leandro MJ, Cambridge G, Edwards JC, Ehrenstein MR, Isenberg DA. B-cell depletion in the treatment of patients with systemic lupus erythematosus: a longitudinal analysis of 24 patients. Rheumatology. 2005;44(12):1542-5.

13. Sfikakis PP, Boletis JN, Tsokos GC. Rituximab anti-B-cell therapy in systemic lupus erythematosus: pointing to the future. Curr Opin Rheumatol. 2005;17(5):550-7.

Avaliação: Editor e dois revisores externos Conflito de interesse: $\mathrm{O}$ autor realizou consultoria médica para a Roche Pharma Produtos Químicos e Farmacêuticos em 2005.

Recebido: 24/10/2006

Aceito após modificações: 09/07/2007 